\title{
Motion Magnification in Solar Imaging Data Sequences in the Sub-pixel Regime
}

\author{
Sihui Zhong ${ }^{1}$ (D) Timothy J. Duckenfield $^{2}$ (D) - Valery M. Nakariakov ${ }^{1,3}$. \\ Sergey A. Anfinogentov ${ }^{4}$
}

Received: 4 June 2021 / Accepted: 21 July 2021 / Published online: 7 September 2021

(C) The Author(s) 2021

\begin{abstract}
The capability of the motion-magnification technique for the detection of transverse oscillations, such as kink oscillations of solar coronal loops observed with an imaging telescope, in the sub-pixel regime is investigated. The technique is applied to artificial-image sequences imitating harmonic transverse displacements of the loop, observed in the optically thin regime. Motion magnification is found to work well on the analysis of sub-pixel, $\geq 0.01$ pixel oscillations, and it is characterised by linear scaling between the magnified amplitude and input amplitude. Oscillations of loops with transverse density profiles of different steepness are considered. After magnification, the original transverse profiles are preserved sufficiently well. The motion-magnification performance is found to be robust in noisy data, for coloured noise with spectral indices ranging from 0 to 3 , and additional Poisson noise with a signal-to-background-noise ratio down to unity. Our findings confirm the reliability of the motion-magnification technique for applications in magnetohydrodynamic seismology of the solar corona.
\end{abstract}

This article belongs to the Topical Collection:

Magnetohydrodynamic (MHD) Waves and Oscillations in the Sun's Corona and MHD Coronal Seismology

Guest Editors: Dmitrii Kolotkov and Bo Li

V.M. Nakariakov

v.nakariakov@warwick.ac.uk

S. Zhong

Sihui.Zhong@warwick.ac.uk

T.J. Duckenfield

tim.duckenfield@kuleuven.be

S.A. Anfinogentov

anfinogentov@iszf.irk.ru

1 Centre for Fusion, Space and Astrophysics, Department of Physics, University of Warwick, Coventry, CV4 7AL, UK

2 Centre for Mathematical Plasma Astrophysics, Department of Mathematics, KU Leuven, Celestijnenlaan 200B bus 2400, 3001 Leuven, Belgium

3 St Petersburg Branch, Special Astrophysical Observatory, Russian Academy of Sciences, St Petersburg 196140, Russia

4 Institute of Solar-Terrestrial Physics SB RAS, Irkutsk 664033, Russia 
Keywords Coronal seismology · Oscillations, solar · Waves, magnetohydrodynamic · Active regions, structure

\section{Introduction}

Magnetohydrodynamic (MHD) wave phenomena in the solar atmosphere remain one of the key research topics in the context of both the coronal heating problem (e.g. Van Doorsselaere et al., 2020) and seismological diagnostics (e.g. Nakariakov and Kolotkov, 2020). An intriguing phenomenon is decayless kink oscillations of coronal loops. Oscillations of this type have first been observed by Wang et al. (2012) with the imaging EUV telescope Atmospheric Imaging Assembly (AIA) onboard the Solar Dynamics Observatory (SDO: Lemen et al., 2012). Similar oscillations are detected as persistent Doppler-shift oscillations at loop apexes (Tian et al., 2012; Li et al., 2018). Anfinogentov, Nisticò, and Nakariakov (2013) demonstrated the ubiquity of decayless kink oscillations of coronal loops, which opens up interesting perspectives for regular seismological probing of the magnetic field in coronal active regions (Anfinogentov and Nakariakov, 2019). Of special interest is the diagnostics of the magnetic-field sigmoidity (Magyar and Nakariakov, 2020), and the related free magnetic energy. The detection of multiple, parallel harmonics provides valuable information about the distribution of physical parameters along the oscillating loop (Duckenfield et al., 2018). In the context of coronal heating, understanding the physical processes in decayless oscillations is of interest for the determination of the coronal-energy balance supported by the input from quasi-stationary or random motions near the footpoints of the oscillating loops; see, e.g., Nakariakov et al. (2016), Afanasyev, Van Doorsselaere, and Nakariakov (2020), Ruderman and Petrukhin (2021), and recent full-MHD numerical simulations (Karampelas and Van Doorsselaere, 2020, 2021; Kohutova and Popovas, 2021; Shi et al., 2021).

The average apparent amplitude of transverse displacements in decayless oscillations has been estimated at about $0.17 \mathrm{Mm}$ with the standard deviation of $0.1 \mathrm{Mm}$ (Anfinogentov, Nakariakov, and Nisticò, 2015). This amplitude is detected at the locations of the highest displacements, while at other segments of the oscillating loop this value is smaller. The plate scale of AIA is 0.6 arcsec pixel ${ }^{-1}$, corresponding to about $0.44 \mathrm{Mm}$ on the solar disk. Also, the spatial resolution of AIA in the $171 \AA$ channel is $1.73 \operatorname{arcsec}$ (Boerner et al., 2012). Thus, the pixel size is about three times bigger than the displacement amplitude of interest, which significantly complicates the analysis of decayless oscillations. This problem has been addressed by developing the technique of Motion Magnification (MM: Anfinogentov and Nakariakov, 2016), acting as a microscope for low-amplitude motions in image sequences (i.e. data cubes or digital videos). The MM-technique advances the method of the Eulerian video magnification designed by $\mathrm{Wu}$ et al. (2012) and Wadhwa et al. (2013). In the MM-technique, an input image sequence is decomposed with the use of the dual tree complex wavelet transform (DTCWT: Selesnick, Baraniuk, and Kingsbury, 2005). Then the temporal phase variations are amplified by a magnification coefficient, which makes subtle oscillatory transverse displacements in the re-synthesised videos visually recognisable. The application of MM allows for a comprehensive study of decayless kink oscillations and their use for MHD seismology. A series of comprehensive tests performed by Anfinogentov and Nakariakov (2016) demonstrated an excellent performance of the MM-technique in the amplification of low-amplitude non-stationary signals, as well as the linear scaling of the output and input signals in a broad range of parameters. The assessment of the MM-performance in the regime of sub-resolution amplitudes is an important next step. 
Another important question is whether the MM-performance is sensitive to the transverse profile of the plasma density; that is, how different profiles can affect the MM outcomes. In the optically thin regime typical for coronal observations, the square of the density profile, integrated along the line-of-sight ( $\mathrm{LoS})$, determines the profile of the observed intensity. This profile is important for understanding the associated thermodynamic processes, and it is crucial for the dynamics of MHD waves (e.g. Van Doorsselaere et al., 2008), including damping by phase-mixing and resonant-absorption mechanisms (e.g. Van Doorsselaere et al., 2020). The shape of the profile, and, in particular, whether it is monolithic or consists of multiple shells or bundles of strands, and with which characteristic scales, remains subject to intensive studies. Brooks, Warren, and Ugarte-Urra (2012) used a combination of spectroscopic and imaging information to conclude that some loops had single monolithic transverse profiles that were fully spatially resolved by AIA, while other loops seem to be composed of a small number of finer, unresolved threads. Higher-resolution images of the corona obtained with the $\mathrm{Hi}$ - $\mathrm{C}$ rocket suggested that typical strand widths could be about $270 \mathrm{~km}$ (Brooks et al., 2013). Aschwanden and Peter (2017) combined observations of $\mathrm{Hi}$ - $\mathrm{C}$ and AIA and concluded that the majority of loops are marginally resolved in AIA images but are fully resolved in $\mathrm{Hi}$ - $\mathrm{C}$ images. Using the observations obtained with $\mathrm{Hi}$ - $\mathrm{C} 2.1$, Williams et al. (2020) estimated the typical width of loops as about $388 \mathrm{~km}$ and $513 \mathrm{~km}$ in cooler and hotter loops, respectively.

The shape of the transverse density profile, especially the steepness of the transition from the maximum to minimum values, received attention too. Aschwanden et al. (2003) demonstrated that the transition is smooth, which is consistent with the observed quality factors of decaying kink oscillations. Pascoe et al. $(2016,2017)$ developed a seismological technique for probing the transverse density profile in a loop performing decaying kink oscillations. Goddard et al. (2017) used observations of 223 loops with AIA to find evidence in favour of smooth profiles, such as with a linear transition-region or Gaussian profile, rather than profiles with infinitely steep boundaries. Goddard, Antolin, and Pascoe (2018) attempted to model and observe the evolution of the transverse density profile caused by the KelvinHelmholtz instability induced by a decaying kink oscillation. A serious shortcoming of the diagnostics based on the use of decaying oscillations is that those techniques cannot give us information before the energy-release events exciting them. This shortcoming is absent from the diagnostics based on decayless kink oscillations that exist before such events. It makes decayless oscillations a promising tool for the diagnostics of the transverse density profile in pre-flaring and pre-eruptive loops. Development of such a seismological method requires understanding the effect of the steepness of the transverse profile on the performance of the MM-technique.

The aim of this study is to consider the performance of the MM-method in the highly sub-resolution regime. The main motivation is to assess the reliability of the MM-outcomes at the oscillating segments sufficiently far from the maximum-amplitude location, which is necessary for the determination of the parallel structure of a kink oscillation and detection of higher parallel harmonics (see, e.g., Duckenfield et al., 2018), and to explore the effect of the steepness of the transverse profile on the MM-performance. In Section 2 we describe the methodology. In Section 3 we present the results obtained. In Section 4 we summarise and discuss the results.

\section{Method}

An artificial-image sequence with five concentric semi-circular loops with enhanced brightness was created in the plane of sky $(\mathrm{PoS})$, i.e. the $x-y$ plane. The simulated loops perform 


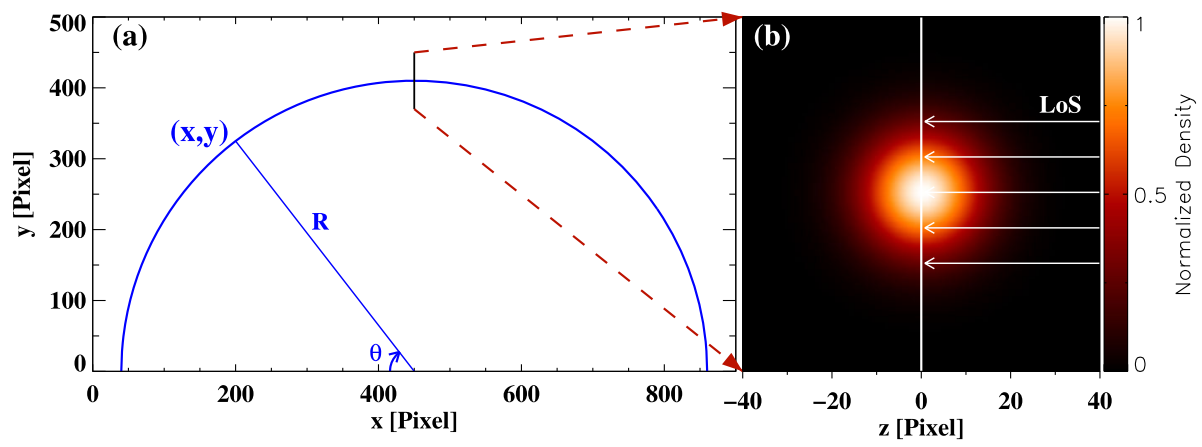

Figure 1 The geometry of an artificial loop. Panel a: The curvilinear axis of the loop in the plane-of-sky ( $x-y$ plane). The major radius of the loop is labelled by $R$. The angle $\theta$ is measured from the $x$-axis in the clockwise direction. The black vertical line indicates the crosssection of loop, displayed in Panel b. Panel b: The density profile across the loop. The white solid arrows show the line-of-sight (LoS). The white vertical line indicates the $x-y$ plane.

decayless oscillatory transverse motions with a sub-pixel amplitude in the PoS. The variation of the oscillation amplitude along the loop corresponds to the fundamental parallel harmonic. The oscillatory displacement of an arbitrary location in a certain loop is

$$
\xi=\xi_{0} \sin \theta \cos \left(\frac{2 \pi t}{P}+\phi\right)
$$

where $\xi_{0}$ is the initial oscillation amplitude, ranging from 0.01 to 0.5 pixel for the innermost and outermost loops, respectively; $\theta$ is the angle measured from the $x$-axis (horizontal direction) in the clockwise direction, see Figure 1a; $P$ is the oscillation period, which is 20 time frames; $t$ is time; and $\phi$ is the initial phase of the oscillation, set to $160^{\circ}$ in this work.

In order to reproduce EUV observations, we construct artificial loops with the transverse density profiles of various steepness. The profiles are described by a generalised Gaussian function with a parameter $p$ that controlled the steepness,

$$
\rho(s)=\rho_{0 p} \exp \left\{-\left(\frac{s^{2}}{2 w^{2}}\right)^{p}\right\} .
$$

Here $s$ is defined as $s^{2}=d^{2}+z^{2}$ where $z \in[-\infty,+\infty]$ is the distance from the PoS in the LoS direction, see Figure $1 \mathrm{~b}$, and $d$ is the distance from a certain pixel to the loop axis in the radial direction in the PoS. The constant $\rho_{0 p}$ is the density at the loop axis for a specific choice of $p$. In addition, the half-width of the Gaussian function $w$ is set to ten pixels to make the transverse profile clearly seen. In this study we are neglecting any time-dependent evolution of the density.

In this work we use the following values of $p: 1,2,5$, and 50, where profiles with a larger value of $p$ are steeper, from a Gaussian at $p=1$ to approaching a step function when $p \rightarrow \infty$. Note that once $p$ varies, the density, mass, and intensity of the loop change correspondingly. Therefore, for a controlled experiment, we impose the condition that loops with different values of $p$ have identical mass. The total mass of a loop crosssection is the integral of the mass of each slice parallel to the LoS. If the cross-sectional mass is constant, the mass of a certain slice should be uniform with different $p$. Thus, we consider this "LoS 

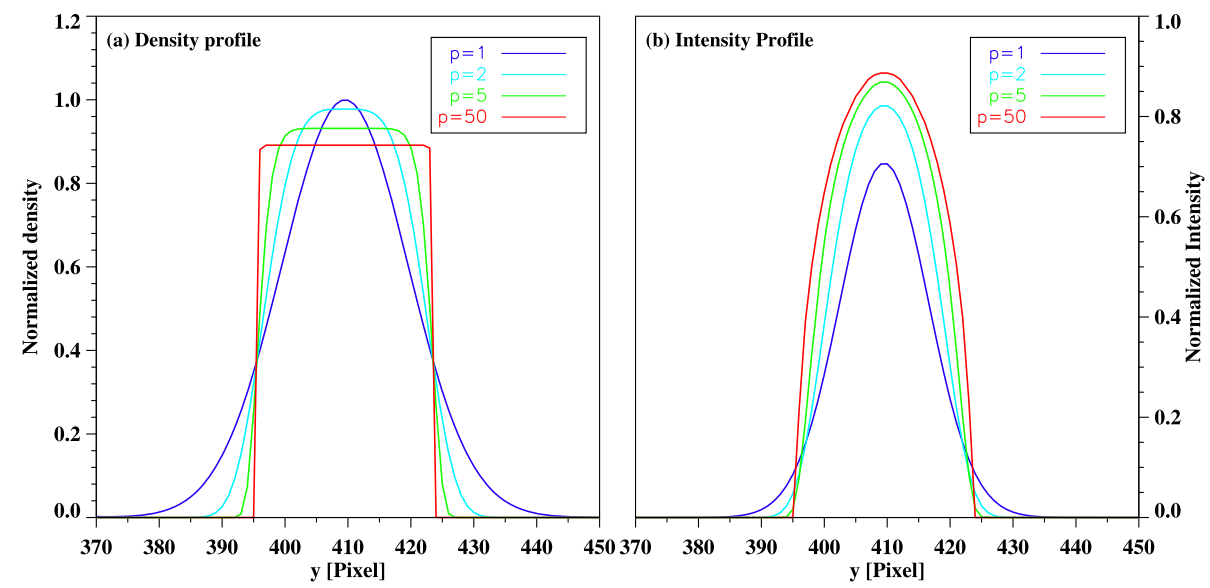

Figure 2 Density profile (Panel a) and the corresponding normalised intensity profile (Panel b) across a loop at the $x-y$ plane for different steepness characterised by the index $[p]$. In Panel a, the area under each curve is the same, meaning that the cross-sectional mass is identical in the loops of different $p$.

mass", which is the integral of the density along the LoS:

$$
\begin{aligned}
\operatorname{mass}_{\mathrm{LoS}} & =\int_{\mathrm{LoS}} \rho_{p} \mathrm{~d} s \\
& =\int_{\mathrm{LoS}} \rho_{0 p} \exp \left\{-\left(\frac{s^{2}}{2 w^{2}}\right)^{p}\right\} \mathrm{d} s \\
& =\int_{-\infty}^{+\infty} \rho_{0 p} \exp \left\{-\left(\frac{d^{2}+z^{2}}{2 w^{2}}\right)^{p}\right\} \mathrm{d} z .
\end{aligned}
$$

To keep mass $\operatorname{LoS}_{\text {S }}$ constant, $\rho_{0 p}$ has different values for different values of $p$. Figure $2 \mathrm{a}$ displays the density profiles across the loop in the PoS for different $p$. The area under each curve, corresponding to the mass, is the same. Assuming these loops to be in thermal equilibrium, the optically thin emission intensity $[I]$ is estimated as an integral of the density squared along the LoS:

$$
\begin{aligned}
I & =\int_{\operatorname{LoS}} \rho_{p}^{2} \mathrm{~d} s \\
& =\int_{-\infty}^{+\infty}\left\{\rho_{0 p} \exp \left(-\left(\frac{d^{2}+z^{2}}{2 w^{2}}\right)\right)^{p}\right) \mathrm{d} z .
\end{aligned}
$$

Figure $2 \mathrm{~b}$ demonstrates the intensity profiles calculated for monolithic density profiles with different steepness $[p]$. It is important to note that a step-function profile of the density does not lead to a step function in intensity. In a more elaborated case, one would need to account for instrumental effects, which we neglect in this study.

In this study, we consider two series of data sets - clear data with the oscillatory signal only, and noisy data (see Section 2.1) - to check whether MM works well in analysis of data with noisy background. These artificial-image sequences are processed with the MMcode described by Anfinogentov and Nakariakov (2016). The smoothing width, which is 
the temporal width of the smoothing filter, is set to be 22 frames, which is $10 \%$ longer than the intended period (20 frames), to avoid introducing a false oscillation. The magnification factor $k$ initially ranges from 3 to 10, which is in the recommended linear scaling range (Anfinogentov and Nakariakov, 2016). Then higher $k$ are considered to find the limitation of the output amplitude. For each value of $k$, we measure the magnified displacement amplitude. It allows us to examine whether the input and output amplitudes scale linearly with each other in the sub-pixel regime. In addition, aiming to test the effect of different transverse profiles on MM, we examine whether the intensity profiles are preserved well after MM. Another question that we address is how the appearance of the loop varies as $p$ grows, which is analysed using the full width at half maximum (FWHM) as a proxy.

\subsection{Noisy Background}

The background brightness in solar EUV image sequences is spatially non-uniform and varies in time. At low frequencies that correspond to periods longer than several minutes, the emission intensity varies as a power law, i.e. it resembles correlated coloured noise. At higher frequencies the noise is white, i.e. uncorrelated (Ireland, McAteer, and Inglis, 2015; Auchère et al., 2016; Kolotkov, Anfinogentov, and Nakariakov, 2016). Referring to this, we include 3D (in $x, y$, and time) background noise as follows: First, 3D Gaussian white noise with the same size as the original data cube is generated. Then uncorrelated noise (high frequencies) is eliminated by a low-pass filter with frequency in the range from 0 to $1 / 150$ (150 is half of the total time frames). Finally, we assign to each pixel a power-law-shaped spectrum with a power-law index $\alpha$ (also called a coloured-noise index): $S=1 / f^{\alpha}$. Here we choose $\alpha=2.5$, having the background vary slowly in time. Then we add background correlated noise and apply Poisson noise to the pure synthetic data, imitating the real EUV image sequences.

\section{Results}

Figure 3 shows an overview of the application of the MM-technique to clear and noisy data sets. The amplitude of the noise is quantified by the signal-to-background-noise ratio (SBR), which equals the ratio of maximum signal intensity and standard deviation of background noise, excluding Poisson noise. In the data set analysed in the right panel, the SBR is 25, and its signal-to-noise ratio (SNR) is $13.24 \mathrm{~dB}$ if the Poisson noise is included. To visualise the oscillatory pattern, we put a vertical slit (the blue line in Figure 3a1) across the loop apexes and make a time-distance map along it. Without MM, the sub-pixel oscillations are not easily seen in the time-distance maps shown in Panels a2 and b2. However, after MM the oscillations are clearly evident; see Panels a3 and b3. Even when the initial amplitude is $\xi_{0}=0.1$ pixels only, it is sufficiently amplified to be recognisable with the chosen magnification factor: $k=10$. Note that there is weak artificial diffusion in the time-distance plots at the maximum and minimum phases of the oscillation, which is discussed in Section 4. As a control test, we processed with MM image sequences without built-in oscillations, i.e. making the displacement $\xi$ in Equation 1 zero, but with the background noise. There are no oscillatory patterns seen in video or time-distance maps of the static loops with the noisy background only.

In order to investigate how the type of the background noise affects the performance of MM, data sets that contained noise with various noise levels and colours were processed; see Figure 4. The Poisson factor remains $5 \%$ in all the tests. The first row of Figure 4 


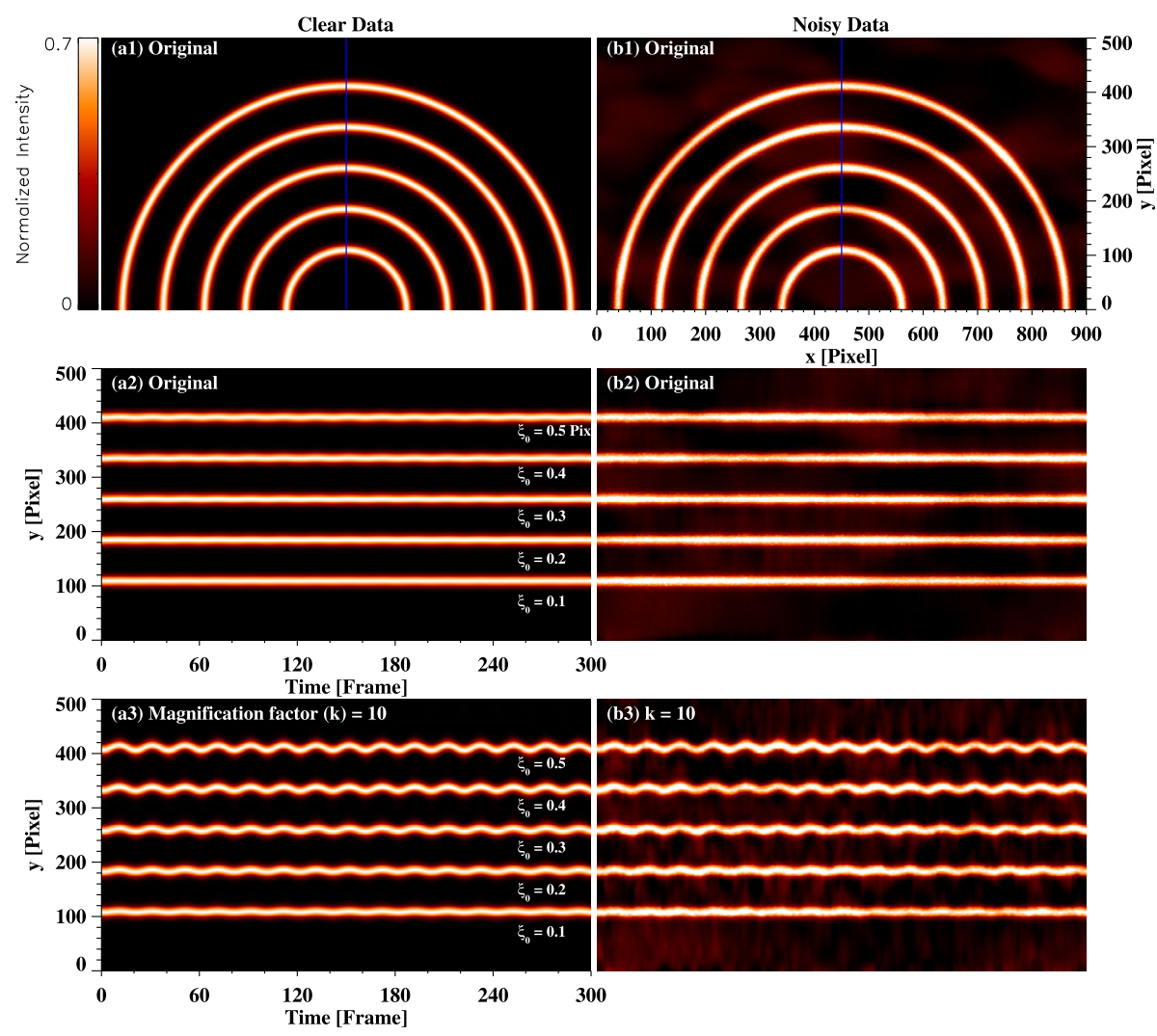

Figure 3 Panels a1-b1: Snapshots of a set of five semi-circular loops undergoing fundamental, decayless kink oscillations in the regime with (right) and without (left) background noise. The oscillation period is 20 time frames. The displacement amplitude $\left[\xi_{0}\right]$ varies from 0.1 to 0.5 pixels, in the inner and outer loops, respectively. The noise is a superposition of correlated coloured noise and Poisson noise. Panels a2-b2: Time-distance maps made along the line indicated by the blue colour in the top panels. The displacement amplitude is indicated. Panels a3-b3: Time-distance maps made with the use of the motion-magnification technique, with the magnification factor $k=10$.

displays noisy images with SBR ranging from ten to one. From Panel a1 to a4, the noise level increases consecutively by a factor around two. The oscillation pattern is recognisable until $\mathrm{SBR}=1$. Beyond this level of the background noise, the oscillation is not easily identified. Thus, for reliable MM-performance, the brightness/intensity contrast between the signal and background noise should exceed the level SBR $>1$. Testing whether a particular type (or colour) of noise adversely affects MM, motion magnification was applied to data sets with different colours of the noise. The second row of Figure 4 shows the results obtained for white noise $(\alpha=0)$, pink noise $(\alpha=1)$, red noise $(\alpha=2)$, and noise with $\alpha=3$. In Panel b1, the background noise is uncorrelated, and the apparent background varies quickly, in contrast to b4 where the background varies slowly and smoothly. For low values of $\alpha, \mathrm{MM}$ introduces some "spikiness" or high spatial wavenumbers into the outcome image, compared to the cases with more correlated noise, although this is unlikely to impact seismological results. 


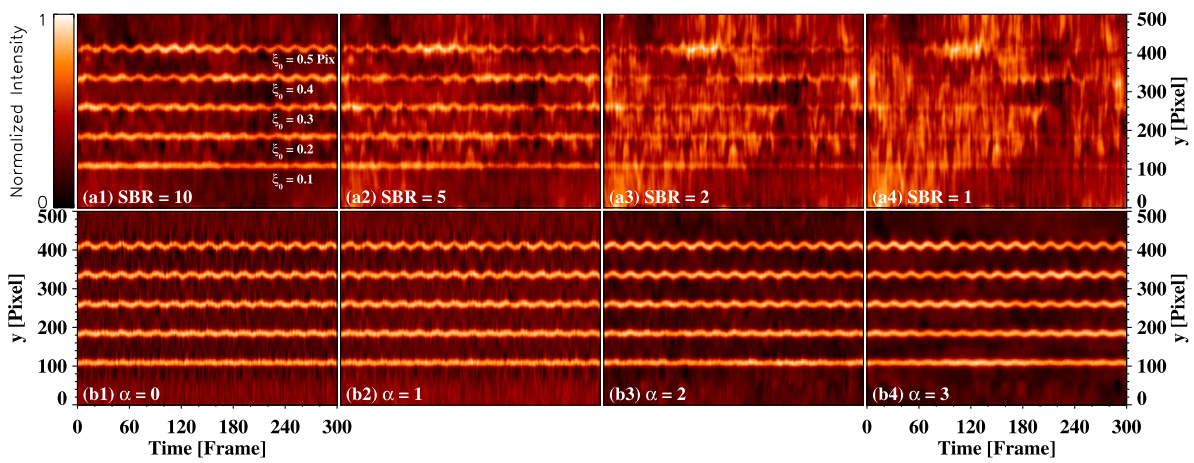

Figure 4 Time-distance maps of magnified data $(k=10)$ with different noise levels (top) and different colour of the noise (bottom). The displacement amplitudes of kink oscillations in the loops are $0.5,0.4,0.3,0.2$, and 0.1 pixels from the top to bottom loops. Intensity values in all the plots are scaled into the range $[0,1]$ for visualisation. In the top row, the signal-to-background-noise ratios are $\mathrm{SBR}=10, \mathrm{SBR}=5, \mathrm{SBR}=2$, $\mathrm{SBR}=1$, from left to right panels. For all top panels, $\alpha=2.5$. In the bottom row, the power-law indices are $\alpha=0$ (white noise), $\alpha=1$ (flicker or pink noise), $\alpha=2$ (red noise), and $\alpha=3$, from left to right panels, respectively. The SBR of Panels b1-b4 is 24.6, 21.6, 24.0, and 23.6, respectively. The Poisson noise in each data set is the same, of $5 \%$. The transverse profile of density is Gaussian.

With the coloured noise (Panels b2-b4) in the background, the oscillation is distinct in all of the analysed cases. The oscillatory pattern is more pronounced if the background evolves more slowly. The reason may be that motion with period longer than the smoothing width will be smoothed. Thus, we conclude that the outcomes of MM are practically independent of the background noise, provided SBR $>1$.

\subsection{Dependence on Input Amplitude}

As described in Section 2, we set the parameters $\xi_{0}$ ranging from 0.1 to 0.5 pixels and $k$ starting with 3 for the test where the transverse density profile is a generalised Gaussian with $p=1$. Here, for brevity, we discuss results of the analysis of a data set with $\xi_{0}=0.3$ pixels as an illustration. The top row of Figure 5 shows time-distance maps of the detected oscillatory pattern. In addition, we compare the transverse intensity profiles obtained at different phases of the oscillations with and without MM in Figure 5a2-b2, respectively. The times for which the intensity profiles are determined cover the whole oscillation cycle, including the maximum, minimum, and a phase in between. Given that in the long-wavelength limit the kink mode is weakly compressive and not significantly distorting the loop's crosssection, the transverse density profile remains unchanged in time in the original data set, so is the intensity profile. In Panel a2 (also see the animations in the Supplementary Materials), the magnified profile has virtually the same shape as the original one at all times. This holds true in the clear data for all values of $k$ and $\xi_{0}$ tested. Even in noisy data, the magnified profiles do not change significantly. That is to say, the loop does not grow narrower or broader in each phase of the oscillation after MM, indicating that MM preserves the profile well for all $k$ and $\xi_{0}$.

Additionally, looking at one test to see the profile's evolution in time (solid curves in Figure 5a2), we see the transverse profile shifts right and then returns to the left, corresponding to the loop oscillating transversely in the PoS. The original profiles almost overlap, because 

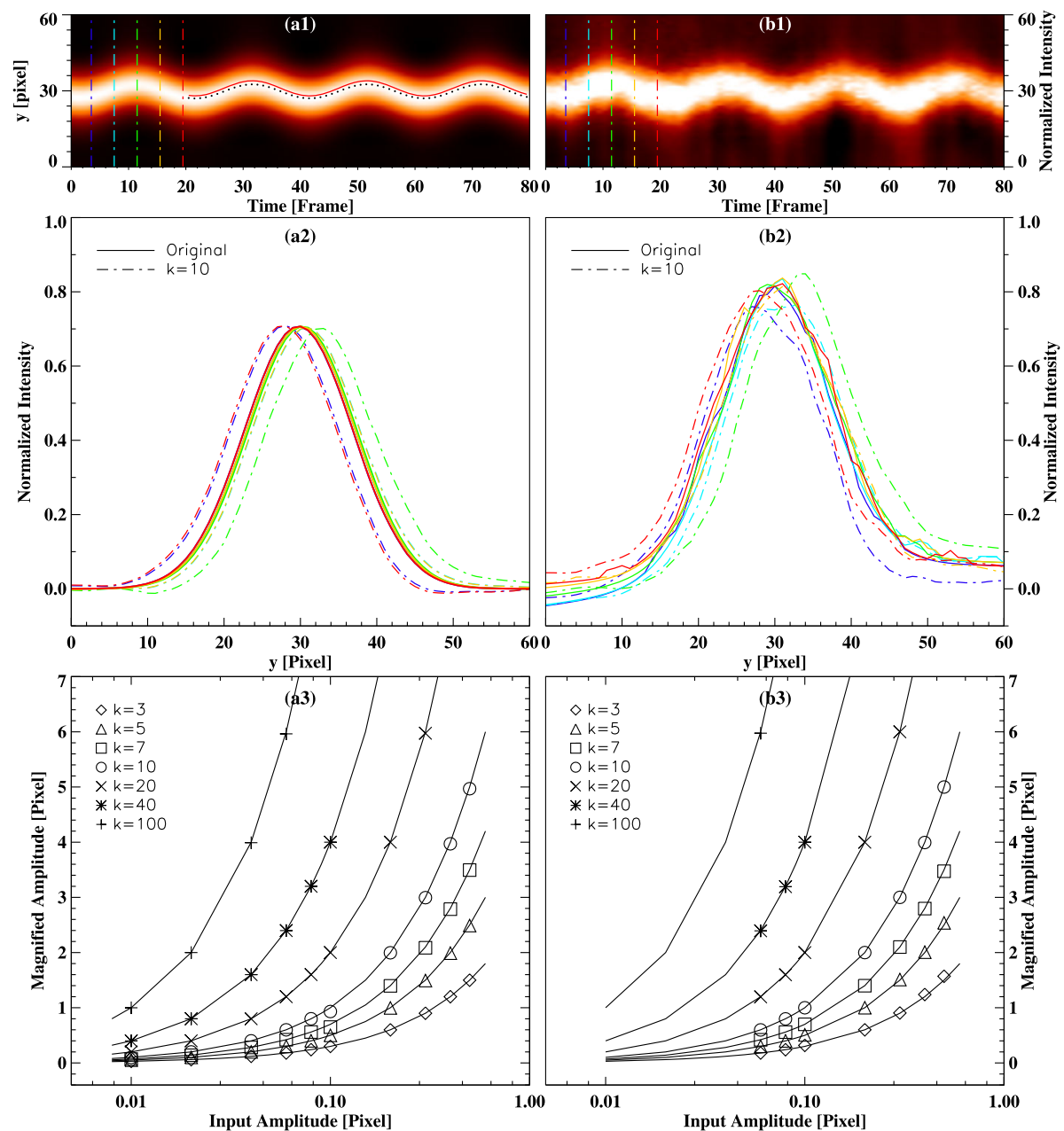

Figure 5 Outcomes of the MM-analysis of an oscillating loop with a Gaussian transverse profile of the density with the displacement amplitude of 0.3 pixels: Time-distance maps for a vertical slit across the apex (Panels a1 and b1); intensity profiles at different phases of the oscillation, shown by dash-dotted lines of the corresponding colour (Panels $\mathbf{a} 2$ and b2); and the scaling of the magnified amplitude with the input amplitude (Panels $\mathbf{a 3}$ and b3), of clear (left column) and noisy data (right column). In Panel a1, the black dots are position of the loop centre obtained from Gaussian fitting of the intensity profile at each instance of time. The red solid curves are the result of the sinusoidal fitting of those positions, slightly offset in the vertical direction. In Panels $\mathbf{a} 2$ and $\mathbf{b 2}$ the solid curves show the profiles obtained from the original data, while the dash-dotted from the outcome of MM. Intensity curves in Panel $\mathbf{b} \mathbf{2}$ were smoothed by a factor of 2. In Panels $\mathbf{a 3}$ and $\mathbf{b 3}$, the black solid curves represent the ideal dependence between input and magnified amplitudes, whose gradient is $3,5,7,10,20,40$, and 100, respectively, corresponding to the magnification factor $3,5,7,10,20,40$, and 100. For the noisy data, input amplitudes less than 0.06 pixels are not shown since for these tiny amplitudes, the variation from fitting the loop's intensity profile (and subsequent fitting of a sinusoid) is too great to reliably extract oscillation parameters after MM.

they shift within \pm 0.3 pixel and the motion offset is too small to be resolved clearly. For the magnified profiles, their motion offsets (relative to the original profile) are evidently detectable, which should be $\xi_{0} \times(k-1)$. 
Fitting the intensity profiles with a Gaussian function (gaussfit.pro in SolarSoftWare), the displacement amplitude variance at the loop centre (denoted by black dots in Figure 5a1) is found. These points are fitted (using curvefit.pro) with a sine function (see also the red solid curve in Figure 5a1 as an illustration):

$$
\xi_{\mathrm{S}}=\xi_{0 \mathrm{~s}} \sin \left(\frac{2 \pi t}{P_{\mathrm{s}}}+\phi_{\mathrm{s}}\right)
$$

All variables in this equation have the same meaning as in Equation 1 but are determined in the magnified data, which is indicated by the additional index S. In particular, $\xi_{0 \text { s }}$, the magnified displacement amplitude, is the key proxy used to compare with the original amplitude $\xi_{0}$. This procedure is applied for all values of $k$. Detailed statistical results are displayed in Table 1 (in the appendix). Recall that in the initial set up (Equation 1), $P$ is 20 frames, $\phi$ is 2.79 radians, and the fitting function is sine not cosine. Therefore, the expected values of $\xi_{0 \mathrm{~s}}$, $P_{\mathrm{s}}, \phi_{\mathrm{s}}$ are $\xi_{0} \times k$ (pixels), 20 (frames), and 4.36 (radians), respectively. In Table 1, for the cases of magnification factors $k=3-10$ most measured parameters satisfy the expectation and errors are less than $10 \%$. In cases of $k \gg 10$, limited by the localisation of the wavelet component in the spatial and wavenumber domains during DTCWT, nonlinear effects come into play when the output amplitude is large, exceeding several pixels (Anfinogentov and Nakariakov, 2016). Generally, the threshold is larger than 20 pixels but it should be checked for specific $k$ and initial input displacement amplitude. This shortcoming does not affect the MM-performance, since in practical applications, an output amplitude of around three to five pixels is enough for the subsequent analysis by traditional methods.

To visualise the main result of MM in the sub-pixel regime, we present the scaling of the measured magnified amplitude with the actual amplitude in Figure 5a3-b3. The magnified amplitudes $\xi_{0 \text { s }}$ obtained for different $k$ are denoted by different symbols. For comparison, we show lines with the gradient of $k$ (black solid curves) indicating the linear scaling relation. All points lie on the linear scaling lines, indicating that the magnified amplitude depends linearly on the input amplitude in the sub-resolution regime.

To figure out in what circumstance MM does not work as expected, we apply the same procedure as above with extremely low amplitudes ranging from 0.01 pixel to 0.08 pixels. Results are included in Figure 5a3-b3 and Figure 6. In the noise-free data, original oscillations with amplitudes as low as 0.01 pixel can be identified. This also indicates that the method that we use for measuring the oscillation amplitude in time-distance maps is capable of measuring amplitudes less than 0.1 pixel. For these cases of extremely low amplitude, a higher magnification factor (e.g. $k>10$ ) should be considered to make the oscillations evident. As mentioned above, there should be a limitation of the output amplitude for each specific $\xi_{0}$ and $k$ to avoid the nonlinear artefact. As our input amplitude $\xi_{0}$ is of the order of 0.01 pixel, we can try $k$ up to 100 . Results show that the input oscillations are successfully revealed by MM, and the magnified oscillation parameters agree with the expected ones; see Figure $5 \mathrm{a} 3$.

However, in the cases of $\xi_{0}<0.06$ in the noisy data $(\mathrm{SBR}=22)$, the oscillation parameters cannot be recovered despite MM. The oscillations are overwhelmed by the noise, significantly affecting the loop fitting so that the extraction of oscillation parameters after $\mathrm{MM}$ is not reliable; see Figure $6 \mathrm{a} 1-\mathrm{a} 2$. When $\xi_{0} \geq 0.06$, the oscillatory pattern in the time-distance plot (see Figure 6b1) is more evident and the confidence of curve fitting (see Figure 6b2) is higher, thus the detection of oscillations after magnification succeeds see Figure 5(b3). Note that, for different noise levels, this threshold amplitude will change - here we simply demonstrate that, even accounting for a reasonable level of noise $(\mathrm{SBR}=22)$, 

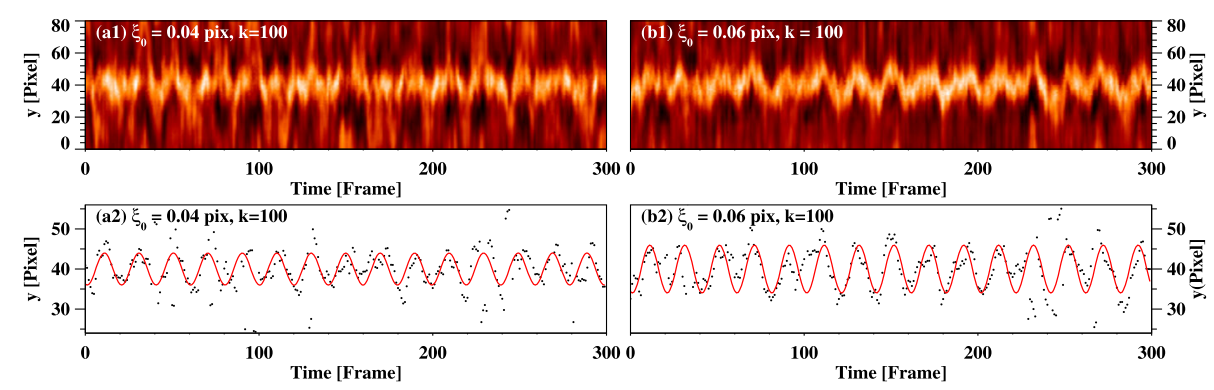

Figure 6 Comparison of the MM-analysis of noisy data with the displacement amplitude of 0.04 and 0.06 pixels: Time-distance maps for a vertical slit across the apex (Panels a1, b1); loop-centre position at each instant of time obtained through Gaussian fitting of intensity profile across the loop and curve fitting of Gaussian fits (Panel a2, b2). In Panel a1, the oscillatory pattern is affected by noise; while in Panel b1 the oscillation is more evident. In Panels $\mathbf{a 2}$ and $\mathbf{b 2}$, the black dots and the red solid curves are similar to those of Figure 5a1 but for the case of $\xi_{0}=0.04$ and $\xi_{0}=0.06$ with $k=100$ of noisy data, respectively.

sub-pixel oscillations can still be extracted with MM. Moreover, practical analysis on real data with oscillation amplitude $<0.1$ pixel is too tricky to consider as it would raise a high level of uncertainty.

To summarise, as long as the transverse motions are "recognisable", i.e. the noise does not conceal the subtle oscillations, measurement of the oscillation parameters after MM will perform perfectly and give a linear scaling of magnified amplitude with input amplitude. In practice, such motions can be found in high-contrast features. Moreover, magnified amplitudes of several pixels are resolvable and sufficient for seismology and other analyses. This value is smaller than the threshold (magnitude of ten pixels), thus the range of $k$ considered in the sub-resolution regime will not lead to nonlinear effects.

\subsection{Effect of Transverse Profile}

Now we apply MM to oscillations of loops with different transverse profiles to determine if these have any effect. Time-distance maps of the original and magnified oscillations are displayed in Figure 7 in the left and middle columns, respectively. In addition, intensity profiles across the loop are extracted for different $k$ and shown in the right column. A loop with the actual displacement amplitude of $\xi_{0}=0.2$ pixel is chosen as representative. Note that loops of the same mass but different transverse profile look different. Combining the time-distance plots (middle column of Figure 7) and intensity profiles (right column of Figure 7), we conclude that the intensity profiles are the same before and after MM. Note that when the loop boundary grows sharper (e.g. $p=50$ ), MM can create a slight artefact near the loop boundaries (see the overshooting outlined in Panel d3), which, however, is below $10 \%$ of maximum intensity and is thus minor.

To test whether MM is sensitive to the profile steepness, we use the full width at half maximum (FWHM) as a proxy of the loop width [ $w]$, because it is less affected by noise than $[w]$. Intensity profiles are fitted with a Gaussian function, and then the FWHM of a certain loop is calculated with the coefficients of the fit at each instant of time. The error is estimated as the standard deviation of all measured FWHM in the time domain. The same process is applied to each case of $p$ and $\xi_{0}$, and to both clear and noisy data. The results are plotted in Figure 8 , with $\xi_{0}=0.1$ and $\xi_{0}=0.3$ pixel being selected as representative. In terms of the original data (see the first column in each panel), the FWHM increases from 16.6 pixel in the case of $p=1$ to 21.6 with $p=50$. The errors are nearly zero in the original data, confirming 


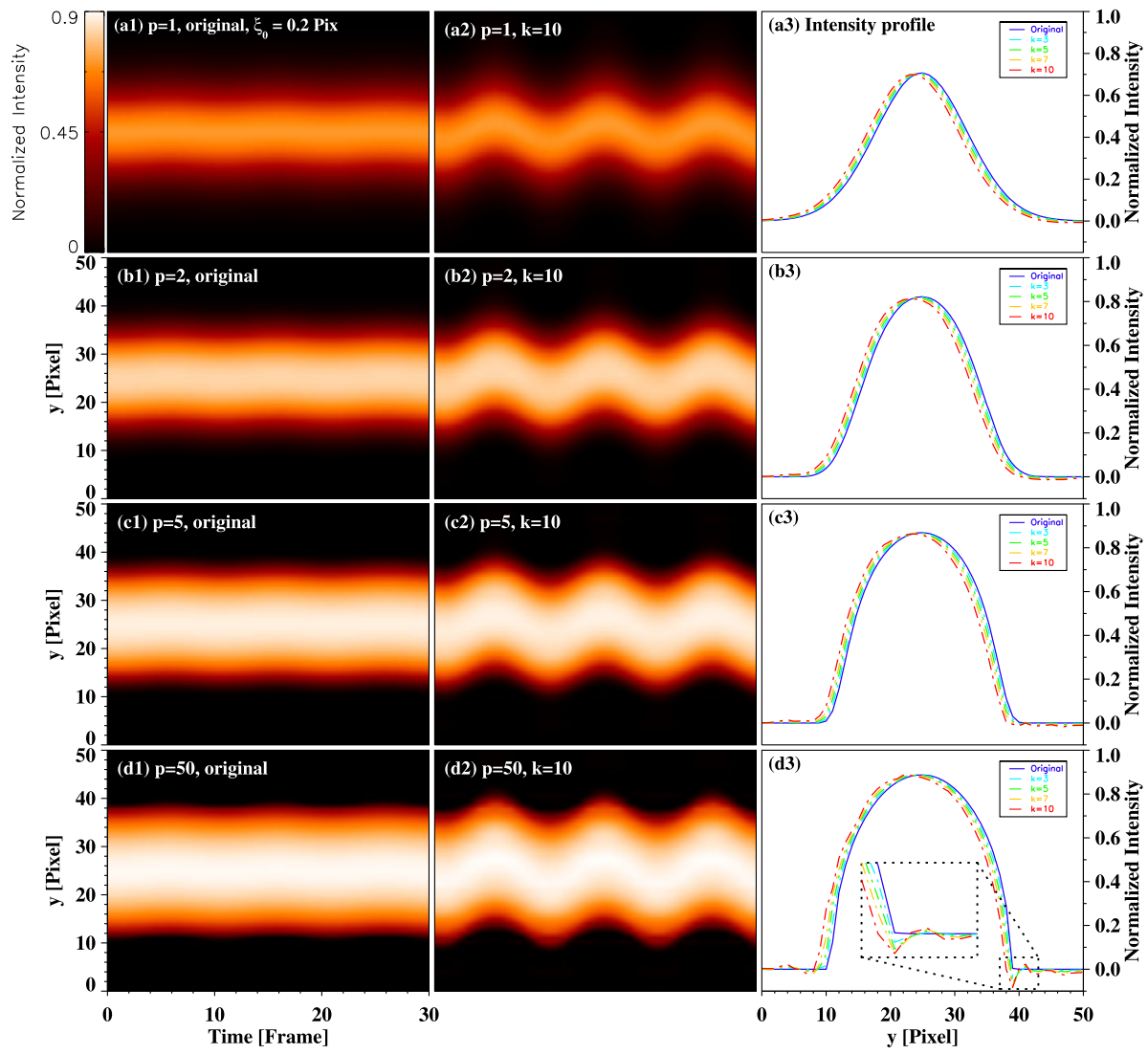

Figure 7 Comparison of detected kink oscillations of loops with different steepness index $p$ before and after MM. The actual displacement amplitude is $\xi_{0}=0.2$ pixel. Panels a1-d1: time-distance maps of original data; Panels a2-d2: time-distance plots of magnified data; Panels a3-d3: intensity profiles extracted from original and magnified data with different magnification coefficient $k$. The blue solid curves are the original intensity profiles, and the coloured dash-dotted curves are the profiles after MM, which are extracted from the corresponding time-distance plots at the same instant of time. In Panel $\mathbf{d 3}$, the "overshoot" artefact is outlined by the small dotted rectangle, whose enlarged version is displayed in the inlets.

that this measurement is reliable. Comparing the FWHM found for different magnification coefficients $k$ and for a fixed $p$, when $k \leq 10$ their differences are so small that they can be ignored, verifying that MM does not change the loop width, as stated before. But in a nonpractical case of $k \gg 10$, FWHM increases gradually because the artefact situated at the loop boundaries become more pronounced as $k$ grows. As expected, errors in the noisy data are bigger than the errors in the pure data. This impact of noise is understandable because i) the magnified evolving background brightness may be comparable to the signal at the loop boundaries, and ii) Poisson noise causes minor enhancement to the inner core of intensity profile, making the profile spiky. The error - that is, the deviation of FWHM - increases as either $k$ or $p$ increases in the noise-free data. This is due to that fact that MM with higher $k$ and $p$ would create more artefact ringing at the sharp gradients at the edge of the loop as mentioned above. Furthermore, in the cases of noisy data, the deviation of FWHM is less than half a pixel in most cases. Even in the cases of the largest error, i.e. in the cases of $p=50$ and $7 \leq k \leq 40$, see Panels a1, a2, and b2, the variance of FWHM is larger than a 


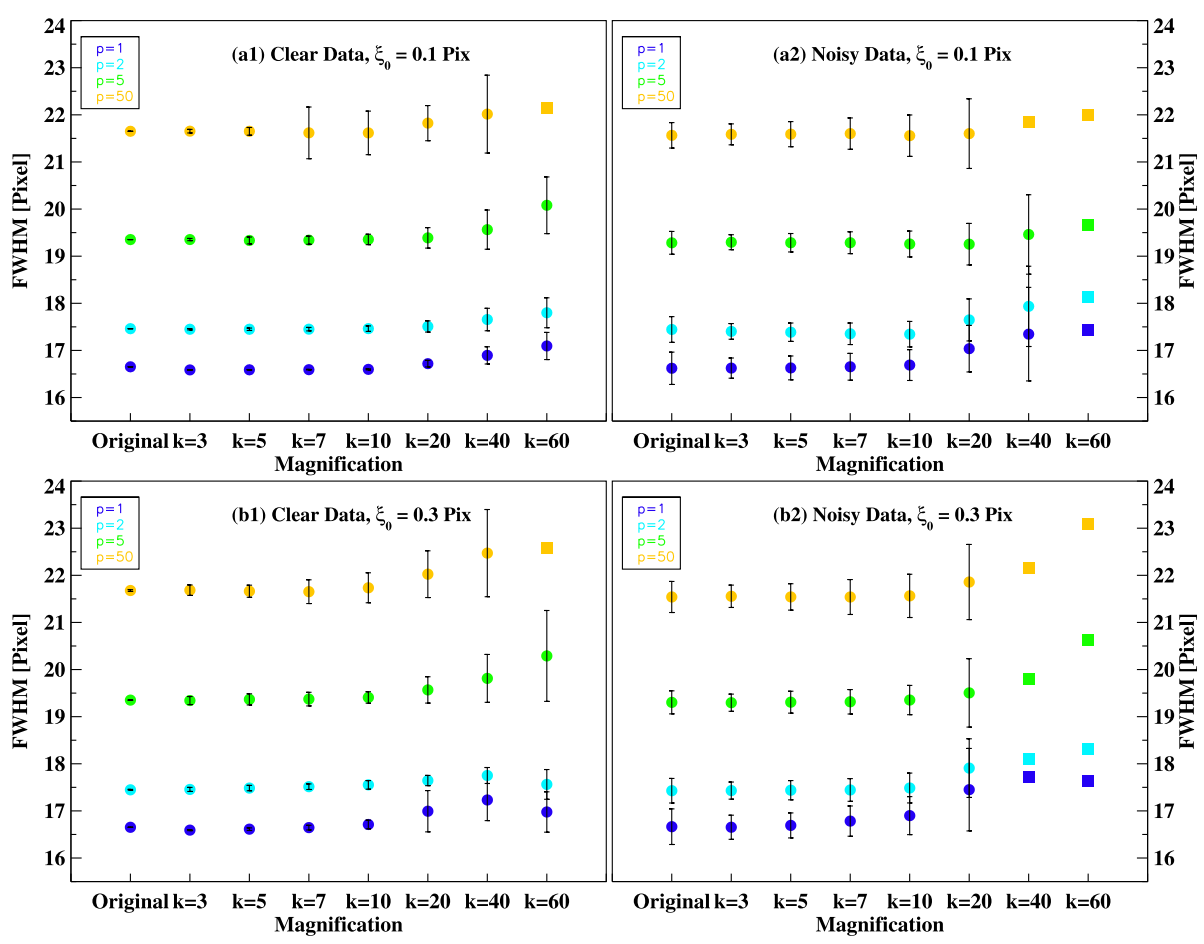

Figure 8 Measured full widths at half maximum (FWHM) of oscillating loops with different steepness indices $p$, which are denoted by different colours. Results obtained for the loops oscillating with the displacement amplitudes $\xi_{0}=0.1,0.3$ pixel are chosen as representative. Panels a1 - b1: clear data; Panels a2 -b2: noisy data. In each case, the original data cube was put through MM with $k$ ranging from 3 to 60 . The measured FWHM are averaged over the time domain, and the error is the standard deviation. Those FWHM with errors greater than unity are denoted by squares rather than circles.

half pixel but less than one pixel. In cases of $k \gg 10$, the error is greater than one pixel, which is not acceptable for standard seismology use.

The MM-technique can cause some artificial diffusion of the loop boundary, especially at the maximum and minimum phases of the oscillation, resulting in varying FWHM of the intensity profile in magnified data. Higher values of the magnification coefficients $k$ may make this artefact more obvious. These artefacts have been pointed out by Anfinogentov and Nakariakov (2016) as "artificial diffusion of the loop crosssection", "artificial cross-influence", or "oscillation leakage". It does not affect the measurements of oscillation amplitudes and periods. To dig deeper into this diffusion-leakage phenomenon, we compare the intensity profiles obtained for different magnification factors $k$ and different steepness indices $p$ in the case of $\xi_{0}=0.3$ pixel in Figure 9. All magnified profiles are extracted at the maximum displacement at the same instant of time, and they are shifted to where the original ones (see black curves in Figure 9) are located, revealing the minor difference between the profiles. The analysis is repeated for the noisy data, with different noise levels of SBR $=25$ in the second row, and SBR $=5$ in the third row. In each panel, the profiles obtained with higher $k$ are seen to gradually deviate from the original profile. The discrepancy increases as the profile turns steeper and the level of noise grows; see the rightmost column and the bottom row as particular examples. Note that the magnified profile with $k=60$ (see the red curves) deviates greatly, losing the original transverse structure. 


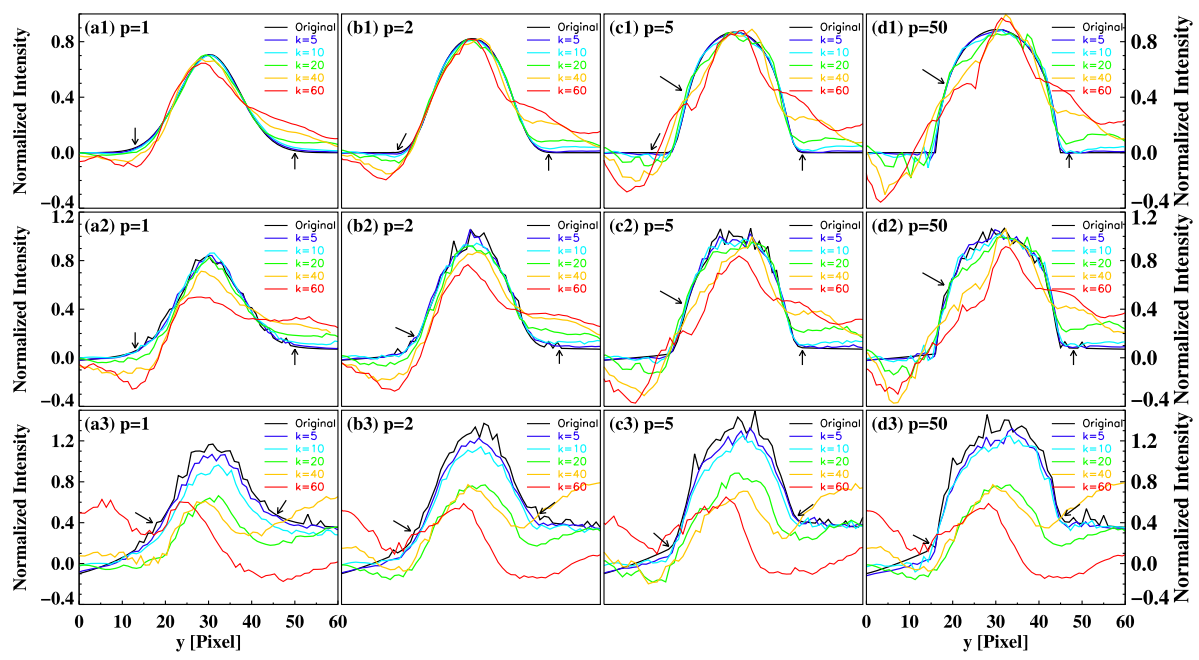

Figure 9 Normalised transverse intensity profiles of kink oscillating loops with different steepness indices $p$, extracted at the maximum displacements. Panels a1-d1: clear data; Panels a2-d2: noisy data with $\mathrm{SBR}=25$; Panels a3 $-\mathbf{d 3}$ : noisy data with $\mathrm{SBR}=5$. Blue solid curves indicate the original profiles. Curves with other colours are the profiles obtained from the magnified data with different magnification coefficient $k$. These magnified profiles are shifted left from the original ones for comparison. The black arrows denote the tendency of how the profiles will be changed after MM.

A proxy $\sigma$ is used in the quantification of difference between the original and magnified profiles. If the profile is perfectly preserved, i.e. the magnified profile is exactly the same as original one, their difference $D$ should be 0 . Thus, $\sigma$ is defined as the deviation of the difference $[D]$ compared to the expected 0: $\sigma=\left(\sum_{i}^{n}\left(D_{i}-0\right)^{2}\right)^{1 / 2} / n$, where $i$ is the pixel index along the profile, and $n$ is the total number of the pixels. Keeping the profile constant at $p=50$, and with $k$ varying from 3 to $60, \sigma$ is found to increase: from 0.038 to 0.231 in clear data; from 0.054 to 0.305 in noisy data with $\mathrm{SBR}=25$; and from 0.081 to 0.653 with $\mathrm{SBR}=5$. The increase in $\sigma$ is manifested as the growing deviation from the original profile in the loop core - see the red curve in Panels $\mathrm{c} 1-\mathrm{d} 2$ and bottom row as particular examples - and is manifested as the subtle gradient increment/decrement of "wings" of these profiles for different $k$, resulting in a "dip" in the left wing and "rise" in the right (denoted by the black arrows in each panel). In the noise-free data set, $\sigma$ in the case of $p=1, p=2, p=5$, $p=50$ with $k=10$ is $0.021,0.036,0.041$, and 0.051 , respectively. In the noisy data with $\mathrm{SBR}=25, \sigma$ in the case of $p=1, p=2, p=5, p=50$ with $k=10$ is $0.047,0.057,0.056$, and 0.073 , respectively. As the profile becomes steeper, i.e. $p$ becomes bigger; see Panels $\mathrm{c} 1$ and $\mathrm{d} 1$, it overshoots at the boundaries. This overshooting is probably due to the finite data length, leading to the DTCWT being imperfect at the large wave numbers needed to reconstruct this edge. Considering $\sigma$ for the cases with $k=20-60$, there are only 5 (out of 12) cases where $\sigma$ is below $10 \%$ of maximum intensity: $p=1$ with $k=20,40$, and $p=2$ with $k=20,40$ in noise-free data and $p=1$ with $k=20$ in noisy data with $\mathrm{SBR}=25$. The $\sigma$ of the remaining cases exceeds the threshold. This indicates that a steeper profile is prone to being distorted more easily, particularly if being magnified with $k>20$. When $k \gg 10$, artefacts added to the two wings of the profile are pronounced enough while the core of the profile remains, hence deviates from the original profile. In addition, a steep edge plays a role in imperfect reconstruction. In noisy data, see the second and third row of Figure 9, the original profiles are spiky while the magnified profiles are somehow smoothed. 
This is likely due to the inherent smoothing of coherent structures when passing through the DTCWT. Generally speaking, a higher magnification factor, steepness, and noise level all result in a larger $\sigma$. In the most cases, $\sigma$ is less than $10 \%$ of the maximum intensity. This indicates that this artefact generated by MM is very minor and below the level of noise when $k \leq 10$ in the case of $\xi_{0}=0.3$ pixel. Even if $k \gg 10$, the information about the oscillation, e.g. the displacement amplitude and period, is preserved.

\section{Summary and Discussion}

In the present work, we investigate the capacity of the MM-technique designed by Anfinogentov and Nakariakov (2016) for the detection of low-amplitude transverse oscillatory displacements of coronal plasma structures, in the sub-pixel regime. We address the scaling of the magnified amplitude with the actual one, and the preservation of the transverse intensity profile across the oscillating loop. The MM-technique is applied to artificial-image sequences imitating kink oscillations of coronal loops, with the oscillation polarisation plane being perpendicular to the LoS, observed in the optically thin regime. The oscillation amplitude ranges from 0.01 to 0.5 pixel. We construct different transverse profiles with the use of a generalised Gaussian function. The parameter $p$ controls the steepness of the profile, while we keep the cross-sectional mass a constant. In imaging observations, loops of the same mass but different steepness have a different apparent loop width. Higher $p$ results in a steeper density and intensity profile. The images are contaminated with noise, which includes both coloured noise with a power-law index ranging from 0 to 3, and Poisson noise. The signal-to-background-noise ratio ranged from 0 to 25 .

The MM-technique is found to work well on the analysis of sub-pixel oscillations, with the amplitude down to as small as 0.01 pixel, giving linear scaling of the magnified amplitude with the input amplitude. This is achieved under the premise of i) the original oscillations being recognisable; ii) the noise level in the original image sequence being less than a certain threshold, i.e. SBR $>1$; iii) the magnification coefficient $k$ and smoothing width $w$ being reasonable, that is the output amplitude should be less than 20 pixels, and $w$ should be longer than the expected oscillation period. Thus, using MM as a microscope, we can resolve oscillations at an arbitrary segment along the loop and, in particular, detect higher parallel, i.e. in the direction along the field, harmonics.

In addition, MM-performance is found to be practically independent of the active background noise if SBR $\geq 1$. This is partly attributed to the approximate shift invariance of DTCWT. The noise that we consider is a small shift in the dominant intensity signal. The noise level indicates the amplitude of the shift (compared to the signal), and the type of coloured noise indicates the scale/frequency of the shift. All these properties of shift would not affect the performance of DTCWT, such that subtle transverse motions in the sub-pixel regime can be captured successfully. Then the noise could be reduced through phase smoothing in the MM-procedure, which is why magnified profiles of the oscillations are smoother than the original spiky profiles in the noisy data shown in Figure 9. Thus, MM can serve as a denoising tool.

After MM, transverse profiles with various steepness are found to be preserved if the magnification factor is not too large ( $k<20$ for the case of $\xi_{0}=0.3$ pixel). However, a rather worse result with a high magnification factor should not be considered as a drawback. 2D wavelets or wavelet-like decompositions always imply a trade-off between spatial and wavenumber resolutions. For MM, the former means ability to resolve different motions in neighbouring structures, while the latter allows for large magnification factors. It is impossible to have both large magnification factors and high spatial resolution (in terms of resolving different motions) at the same time. Since in EUV images of the Sun, there 
are a lot of apparently overlapping loops, in the solar context high spatial resolution is more important than ability to use high magnification coefficients. Therefore, in the MMimplementation developed by Anfinogentov and Nakariakov (2016) the balance has been shifted towards spatial resolution. Additionally, we need to stress that, for the majority of seismological applications, large values of the magnification coefficient $(k>10$ for example) are not usually practical or warranted, so this result does not put any real limitations on the MM-performance. A small visual artefact caused by MM, discussed in Section 3.2 is very minor and should not have a significant impact on the result. It makes seismological diagnostics based on MM-processed data possible to probe the density/intensity profiles of the oscillating structure. It would allow one to validate various models of the transverse profiles in various oscillating non-uniformities such as coronal loops, streamers, and current sheets (see, e.g., Martens, 2010; Kolotkov, Vasko, and Nakariakov, 2015; Cargill, De Moortel, and Kiddie, 2016; Pascoe, Hood, and Van Doorsselaere, 2019). Moreover, it would allow us to study the evolution of this transverse structuring that is associated with a specific mechanism of the oscillation dissipation (e.g. Goddard, Antolin, and Pascoe, 2018). For example, to account for the energy supply and losses in decayless kink oscillations, we can make oscillation amplitude and density profile evolve simultaneously, in terms of self-oscillatory model (Nakariakov et al., 2016), i.e. these two properties are controlled by a single parameter or random-driver model (Afanasyev, Van Doorsselaere, and Nakariakov, 2020). In this way, investigation of these simulations can shed light on the physical nature of decayless kink oscillations.

Overall, MM only amplifies transverse motions, preserving other properties/information such as the intensity variation in the time domain. To be more specific, the first several steps of the MM-procedure, i.e. the spatial decomposition and phase computation, identify motions (the phase variation at each pixel) only, and at the end the desired magnified motions are well reconstructed into the final 3D data cube, without producing a significant false signal. Those findings confirm the robustness of the motion-magnification technique for applications in MHD coronal seismology.

In the near future, the highest spatial resolution of the remote-sensing imagers is still of the order of hundreds of $\mathrm{km}$. For instance, the pixel size of the Extreme Ultraviolet Imager (Rochus et al., 2020) onboard the Solar Orbiter (Müller et al., 2020) is about 0.5 arcsec, that is $363 \mathrm{~km}$, two times larger than mean amplitude of decayless kink oscillation $(170 \mathrm{~km})$. Thus, $\mathrm{MM}$ has a promising potential in a wide range of applications in analysing small-scale features or events from upcoming instruments.

\section{Appendix A: Additional Table}

Table 1 Comparison of the kink-oscillation parameters in the original data set and processed with MM.

\begin{tabular}{|c|c|c|c|c|c|c|c|}
\hline \multirow[t]{2}{*}{$k$} & \multirow[t]{2}{*}{$\xi_{0}[$ pixel] } & \multicolumn{3}{|l|}{ Clear data } & \multicolumn{3}{|l|}{ Noisy data } \\
\hline & & $\xi_{0 \mathrm{~s}}$ [pixel] & $P_{\mathrm{S}}[$ frame $]$ & $\phi_{s}[$ radian] & $\xi_{0 \text { s }}$ [pixel] & $P_{\mathrm{S}}[$ frame $]$ & $\phi_{\mathrm{S}}$ [radian] \\
\hline \multirow[t]{5}{*}{3} & 0.1 & $0.30 \pm 0.00$ & $20.00_{-0.37}^{+0.37}$ & $4.36 \pm 0.05$ & $0.32 \pm 0.00$ & $19.99_{-0.62}^{+0.66}$ & $4.36 \pm 0.00$ \\
\hline & 0.2 & $0.60 \pm 0.00$ & $20.00_{-0.34}^{+0.37}$ & $4.36 \pm 0.09$ & $0.60 \pm 0.00$ & $20.01_{-0.60}^{+0.66}$ & $4.36 \pm 0.01$ \\
\hline & 0.3 & $0.90 \pm 0.00$ & $20.00_{-0.36}^{+0.37}$ & $4.37 \pm 0.00$ & $0.91 \pm 0.01$ & $20.03_{-1.85}^{+2.27}$ & $4.36 \pm 0.01$ \\
\hline & 0.4 & $1.20 \pm 0.00$ & $20.00_{-0.35}^{+0.37}$ & $4.36 \pm 0.00$ & $1.24 \pm 0.01$ & $19.98_{-1.63}^{+1.95}$ & $4.36 \pm 0.01$ \\
\hline & 0.5 & $1.50 \pm 0.00$ & $20.00_{-0.36}^{+0.37}$ & $4.36 \pm 0.00$ & $1.57 \pm 0.01$ & $20.02_{-1.64}^{+1.96}$ & $4.36 \pm 0.01$ \\
\hline
\end{tabular}


Table 1 (Continued)

\begin{tabular}{|c|c|c|c|c|c|c|c|}
\hline \multirow[t]{2}{*}{$k$} & \multirow[t]{2}{*}{$\xi_{0}[$ pixel] } & \multicolumn{3}{|l|}{ Clear data } & \multicolumn{3}{|l|}{ Noisy data } \\
\hline & & $\xi_{0 \mathrm{~s}}[$ pixel] & $P_{\mathrm{S}}[$ frame $]$ & $\phi_{s}$ [radian] & $\xi_{0 \text { s }}$ [pixel] & $P_{\mathrm{S}}[$ frame $]$ & $\phi_{\mathrm{S}}[$ radian] \\
\hline \multirow[t]{5}{*}{5} & 0.1 & $0.50 \pm 0.00$ & $20.00_{-1.03}^{+1.14}$ & $4.36 \pm 0.01$ & $0.51 \pm 0.00$ & $20.00_{-3.42}^{+0.52}$ & $4.36 \pm 0.00$ \\
\hline & 0.2 & $1.00 \pm 0.02$ & $\begin{array}{r}20.00_{-2.84}^{+3.97} \\
\text { r }\end{array}$ & $4.36 \pm 0.02$ & $1.00 \pm 0.02$ & $\begin{array}{r}20.02_{-2.84}^{+3.97} \\
\end{array}$ & $4.36 \pm 0.02$ \\
\hline & 0.3 & $1.49 \pm 0.02$ & $20.00_{-2.00}^{+2.51}$ & $4.36 \pm 0.01$ & $1.51 \pm 0.02$ & $20.00_{-1.95}^{+2.43}$ & $4.36 \pm 0.01$ \\
\hline & 0.4 & $1.99 \pm 0.02$ & $20.00_{-1.53}^{+1.80}$ & $4.36 \pm 0.01$ & $2.01 \pm 0.02$ & $20.00_{-1.53}^{+1.81}$ & $4.36 \pm 0.01$ \\
\hline & 0.5 & $2.49 \pm 0.02$ & $20.00_{-1.25}^{+1.43}$ & $4.36 \pm 0.01$ & $2.54 \pm 0.05$ & $\begin{array}{r}19.99_{-3.42}^{+5.21} \\
-3\end{array}$ & $4.36 \pm 0.02$ \\
\hline \multirow[t]{5}{*}{7} & 0.1 & $0.65 \pm 0.00$ & $20.00_{-0.13}^{+0.13}$ & $4.36 \pm 0.00$ & $0.70 \pm 0.00$ & $19.99_{-0.40}^{+0.42}$ & $4.36 \pm 0.00$ \\
\hline & 0.2 & $1.40 \pm 0.01$ & $19.99_{-1.03}^{+1.14}$ & $4.36 \pm 0.01$ & $1.40 \pm 0.00$ & $20.00_{-0.39}^{+0.40}$ & $4.36 \pm 0.00$ \\
\hline & 0.3 & $2.09 \pm 0.01$ & $19.99_{-1.03}^{+1.14}$ & $4.36 \pm 0.01$ & $2.10 \pm 0.00$ & $20.00_{-0.37}^{+0.38}$ & $4.36 \pm 0.00$ \\
\hline & 0.4 & $2.78 \pm 0.01$ & $19.98_{-1.03}^{+1.14}$ & $4.36 \pm 0.01$ & $2.80 \pm 0.01$ & $20.00_{-0.34}^{+0.36}$ & $4.36 \pm 0.00$ \\
\hline & 0.5 & $3.49 \pm 0.02$ & $20.02_{-1.03}^{+1.15}$ & $4.36 \pm 0.01$ & $3.47 \pm 0.01$ & $20.00_{-0.35}^{+0.36}$ & $4.36 \pm 0.00$ \\
\hline \multirow[t]{5}{*}{10} & 0.1 & $0.99 \pm 0.02$ & $20.00_{-2.89}^{+4.06}$ & $4.36 \pm 0.02$ & $1.00 \pm 0.02$ & $20.00_{-1.56}^{+1.81}$ & $4.36 \pm 0.02$ \\
\hline & 0.2 & $1.99 \pm 0.01$ & $\begin{array}{r}19.99_{-1.02}^{+1.14} \\
\end{array}$ & $4.36 \pm 0.01$ & $2.00 \pm 0.02$ & $20.01_{-0.06}^{+0.06}$ & $4.36 \pm 0.01$ \\
\hline & 0.3 & $2.98 \pm 0.02$ & $\begin{array}{r}19.99_{-1.08}^{+1.22} \\
\end{array}$ & $4.36 \pm 0.01$ & $3.00 \pm 0.00$ & $20.00_{-0.06}^{+0.06}$ & $4.36 \pm 0.00$ \\
\hline & 0.4 & $3.97 \pm 0.05$ & $19.99_{-2.31}^{+3.00}$ & $4.36 \pm 0.01$ & $3.99 \pm 0.02$ & $20.01_{-0.80}^{+0.86}$ & $4.36 \pm 0.00$ \\
\hline & 0.5 & $4.98 \pm 0.02$ & $\begin{array}{r}19.99_{-0.62}^{+0.66} \\
-0.62\end{array}$ & $4.36 \pm 0.00$ & $5.00 \pm 0.02$ & $20.00_{-0.62}^{+0.66}$ & $4.36 \pm 0.00$ \\
\hline \multirow[t]{5}{*}{20} & 0.1 & $2.00 \pm 0.00$ & $20.00_{-0.35}^{+0.36}$ & $4.36 \pm 0.00$ & $2.00 \pm 0.00$ & $20.00_{-0.37}^{+0.39}$ & $4.36 \pm 0.00$ \\
\hline & 0.2 & $4.00 \pm 0.01$ & $20.00_{-0.35}^{+0.36}$ & $4.36 \pm 0.00$ & $4.00 \pm 0.01$ & $20.01_{-0.36}^{+0.38}$ & $4.36 \pm 0.00$ \\
\hline & 0.3 & $5.98 \pm 0.03$ & $20.01_{-1.04}^{+1.16}$ & $4.36 \pm 0.01$ & $6.00 \pm 0.00$ & $20.00_{-0.01}^{+0.01}$ & $4.36 \pm 0.00$ \\
\hline & 0.4 & $7.93 \pm 0.04$ & $\begin{array}{r}19.99_{-1.02}^{+1.14} \\
\text { r }\end{array}$ & $4.36 \pm 0.01$ & $7.99 \pm 0.01$ & $20.01_{-0.33}^{+0.34}$ & $4.36 \pm 0.00$ \\
\hline & 0.5 & $10.00 \pm 0.02$ & $20.00_{-0.35}^{+0.36}$ & $4.36 \pm 0.00$ & $10.00 \pm 0.02$ & $20.00_{-0.33}^{+0.34}$ & $4.36 \pm 0.00$ \\
\hline \multirow[t]{5}{*}{40} & 0.1 & $4.00 \pm 0.01$ & $20.00_{-0.35}^{+0.36}$ & $4.36 \pm 0.01$ & $4.00 \pm 0.01$ & $19.99_{-0.37}^{+0.39}$ & $4.36 \pm 0.00$ \\
\hline & 0.2 & $8.00 \pm 0.01$ & $20.00_{-0.35}^{+0.36}$ & $4.36 \pm 0.00$ & $8.00 \pm 0.00$ & $20.01_{-0.11}^{+0.11}$ & $4.36 \pm 0.00$ \\
\hline & 0.3 & $11.90 \pm 0.02$ & $20.00_{-0.34}^{+0.35}$ & $4.36 \pm 0.00$ & $12.00 \pm 0.02$ & $20.00_{-0.33}^{+0.34}$ & $4.36 \pm 0.00$ \\
\hline & 0.4 & $15.87 \pm 0.08$ & $19.99_{-0.99}^{+1.10}$ & $4.36 \pm 0.01$ & $15.96 \pm 0.02$ & $20.01_{-0.31}^{+0.32}$ & $4.36 \pm 0.00$ \\
\hline & 0.5 & $20.00 \pm 0.03$ & $20.00_{-0.34}^{+0.37}$ & $4.36 \pm 0.00$ & $20.00 \pm 0.03$ & $20.00_{-0.32}^{+0.33}$ & $4.36 \pm 0.00$ \\
\hline \multirow[t]{5}{*}{60} & 0.1 & $6.00 \pm 0.01$ & $20.00_{-0.35}^{+0.36}$ & $4.36 \pm 0.00$ & $6.00 \pm 0.01$ & $19.99_{-0.37}^{+0.38}$ & $4.36 \pm 0.00$ \\
\hline & 0.2 & $12.00 \pm 0.02$ & $20.00_{-0.35}^{+0.36}$ & $4.36 \pm 0.00$ & $11.97 \pm 0.00$ & $20.01_{-0.01}^{+0.01}$ & $4.36 \pm 0.00$ \\
\hline & 0.3 & $17.91 \pm 0.09$ & $19.99_{-1.00}^{+1.11}$ & $4.36 \pm 0.01$ & $17.48 \pm 0.03$ & $20.04_{-0.44}^{+0.48}$ & $4.36 \pm 0.00$ \\
\hline & 0.4 & $23.86 \pm 0.12$ & $\begin{array}{r}19.99_{-0.95}^{+1.05} \\
\text { r }\end{array}$ & $4.36 \pm 0.01$ & $23.59 \pm 0.10$ & $19.98_{-0.85}^{+0.93}$ & $4.36 \pm 0.00$ \\
\hline & 0.5 & $29.38 \pm 0.05$ & $20.00_{-0.33}^{+0.34}$ & $4.36 \pm 0.00$ & $29.39 \pm 0.13$ & $20.02_{-0.85}^{+0.93}$ & $4.36 \pm 0.00$ \\
\hline
\end{tabular}


Table 1 (Continued)

\begin{tabular}{|c|c|c|c|c|c|c|c|}
\hline \multirow[t]{2}{*}{$k$} & \multirow[t]{2}{*}{$\xi_{0}$ [pixel] } & \multicolumn{3}{|l|}{ Clear data } & \multicolumn{3}{|l|}{ Noisy data } \\
\hline & & $\xi_{0 \mathrm{~s}}[$ pixel] & $P_{\mathrm{S}}[$ frame $]$ & $\phi_{s}[$ radian] & $\xi_{0 \text { s }}[$ pixel] & $P_{\mathrm{S}}[$ frame $]$ & $\phi_{\mathrm{S}}[$ radian] \\
\hline \multirow[t]{5}{*}{80} & 0.1 & $7.98 \pm 0.01$ & $20.00_{-0.34}^{+0.36}$ & $4.36 \pm 0.00$ & $8.00 \pm 0.02$ & $19.98_{-0.37}^{+0.39}$ & $4.36 \pm 0.00$ \\
\hline & 0.2 & $16.00 \pm 0.03$ & $20.00_{-0.35}^{+0.36}$ & $4.36 \pm 0.00$ & $16.00 \pm 0.01$ & $20.00_{-0.00}^{+0.00}$ & $4.36 \pm 0.00$ \\
\hline & 0.3 & $23.86 \pm 0.12$ & $19.99_{-0.96}^{+1.06}$ & $4.36 \pm 0.00$ & $23.82 \pm 0.09$ & $19.94_{-0.73}^{+0.79}$ & $4.36 \pm 0.00$ \\
\hline & 0.4 & \multicolumn{6}{|c|}{ MOTION MAGNIFICATION FAILS, FITTING INVALID } \\
\hline & 0.5 & \multicolumn{6}{|c|}{ MOTION MAGNIFICATION FAILS, FITTING INVALID } \\
\hline \multirow[t]{5}{*}{100} & 0.1 & $9.93 \pm 0.02$ & $20.00_{-0.35}^{+0.36}$ & $4.36 \pm 0.00$ & $9.98 \pm 0.02$ & $20.00_{-0.37}^{+0.39}$ & $4.36 \pm 0.00$ \\
\hline & 0.2 & $20.00 \pm 0.03$ & $20.00_{-0.35}^{+0.36}$ & $4.36 \pm 0.00$ & $5.05 \pm 0.13$ & $18.56_{-3.55}^{+5.74}$ & $4.36 \pm 0.03$ \\
\hline & 0.3 & \multicolumn{6}{|c|}{ FITTING INVALID } \\
\hline & 0.4 & \multicolumn{6}{|c|}{ FITTING INVALID } \\
\hline & 0.5 & \multicolumn{6}{|c|}{ FITTING INVALID } \\
\hline
\end{tabular}

Supplementary Information The online version contains supplementary material available at https://doi.org/ 10.1007/s11207-021-01870-w.

Acknowledgements S. Zhong was supported by China Scholarship Council-University of Warwick Scholarship 20-21. T.J. Duckenfield was supported by the European Research Council (ERC) under the European Union's Horizon 2020 research and innovation programme (grant agreement No. 724326). V.M. Nakariakov and A.S. Anfinogentov (the problem formulation, interpretations and validation of results) were supported by Russian Scientific Foundation grant 21-12-00195.

\section{Declarations}

Disclosure of Potential Conflicts of Interests The authors declare that they have no conflicts of interest.

Open Access This article is licensed under a Creative Commons Attribution 4.0 International License, which permits use, sharing, adaptation, distribution and reproduction in any medium or format, as long as you give appropriate credit to the original author(s) and the source, provide a link to the Creative Commons licence, and indicate if changes were made. The images or other third party material in this article are included in the article's Creative Commons licence, unless indicated otherwise in a credit line to the material. If material is not included in the article's Creative Commons licence and your intended use is not permitted by statutory regulation or exceeds the permitted use, you will need to obtain permission directly from the copyright holder. To view a copy of this licence, visit http://creativecommons.org/licenses/by/4.0/.

\section{References}

Afanasyev, A.N., Van Doorsselaere, T., Nakariakov, V.M.: 2020, Excitation of decay-less transverse oscillations of coronal loops by random motions. Astron. Astrophys. 633, L8. DOI. ADS.

Anfinogentov, S., Nakariakov, V.M.: 2016, Motion magnification in coronal seismology. Solar Phys. 291, 3251. DOI. ADS.

Anfinogentov, S.A., Nakariakov, V.M.: 2019, Magnetohydrodynamic seismology of quiet solar active regions. Astrophys. J. Lett. 884, L40. DOI. ADS.

Anfinogentov, S.A., Nakariakov, V.M., Nisticò, G.: 2015, Decayless low-amplitude kink oscillations: a common phenomenon in the solar corona? Astron. Astrophys. 583, A136. DOI. ADS. 
Anfinogentov, S., Nisticò, G., Nakariakov, V.M.: 2013, Decay-less kink oscillations in coronal loops. Astron. Astrophys. 560, A107. DOI. ADS.

Aschwanden, M.J., Peter, H.: 2017, The width distribution of loops and strands in the solar corona-are we hitting rock bottom? Astrophys. J. 840, 4. DOI. ADS.

Aschwanden, M.J., Nightingale, R.W., Andries, J., Goossens, M., Van Doorsselaere, T.: 2003, Observational tests of damping by resonant absorption in coronal loop oscillations. Astrophys. J. 598, 1375. DOI. ADS.

Auchère, F., Froment, C., Bocchialini, K., Buchlin, E., Solomon, J.: 2016, On the Fourier and wavelet analysis of coronal time series. Astrophys. J. 825, 110. DOI. ADS.

Boerner, P., Edwards, C., Lemen, J., Rausch, A., Schrijver, C., Shine, R., Shing, L., Stern, R., Tarbell, T., Title, A., Wolfson, C.J., Soufli, R., Spiller, E., Gullikson, E., McKenzie, D., Windt, D., Golub, L., Podgorski, W., Testa, P., Weber, M.: 2012, Initial calibration of the Atmospheric Imaging Assembly (AIA) on the Solar Dynamics Observatory (SDO). Solar Phys. 275, 41. DOI. ADS.

Brooks, D.H., Warren, H.P., Ugarte-Urra, I.: 2012, Solar coronal loops resolved by Hinode and the Solar Dynamics Observatory. Astrophys. J. Lett. 755, L33. DOI. ADS.

Brooks, D.H., Warren, H.P., Ugarte-Urra, I., Winebarger, A.R.: 2013, High spatial resolution observations of loops in the solar corona. Astrophys. J. Lett. 772, L19. DOI. ADS.

Cargill, P.J., De Moortel, I., Kiddie, G.: 2016, Coronal density structure and its role in wave damping in loops. Astrophys. J. 823, 31. DOI. ADS.

Duckenfield, T., Anfinogentov, S.A., Pascoe, D.J., Nakariakov, V.M.: 2018, Detection of the second harmonic of decay-less kink oscillations in the solar corona. Astrophys. J. Lett. 854, L5. DOI. ADS.

Goddard, C.R., Antolin, P., Pascoe, D.J.: 2018, Evolution of the transverse density structure of oscillating coronal loops inferred by forward modeling of EUV intensity. Astrophys. J. 863, 167. DOI. ADS.

Goddard, C.R., Pascoe, D.J., Anfinogentov, S., Nakariakov, V.M.: 2017, A statistical study of the inferred transverse density profile of coronal loop threads observed with SDO/AIA. Astron. Astrophys. 605, A65. DOI. ADS.

Ireland, J., McAteer, R.T.J., Inglis, A.R.: 2015, Coronal Fourier power spectra: implications for coronal seismology and coronal heating. Astrophys. J. 798, 1. DOI. ADS.

Karampelas, K., Van Doorsselaere, T.: 2020, Generating transverse loop oscillations through a steady-flow driver. Astrophys. J. Lett. 897, L35. DOI. ADS.

Karampelas, K., Van Doorsselaere, T.: 2021, Transverse loop oscillations via vortex shedding: a selfoscillating process. Astrophys. J. Lett. 908, L7. DOI. ADS.

Kohutova, P., Popovas, A.: 2021, Excitation and evolution of coronal oscillations in self-consistent 3D radiative MHD simulations of the solar atmosphere. Astron. Astrophys. 647, A81. DOI. ADS.

Kolotkov, D.Y., Anfinogentov, S.A., Nakariakov, V.M.: 2016, Empirical mode decomposition analysis of random processes in the solar atmosphere. Astron. Astrophys. 592, A153. DOI. ADS.

Kolotkov, D.Y., Vasko, I.Y., Nakariakov, V.M.: 2015, Kinetic model of force-free current sheets with nonuniform temperature. Phys. Plasmas 22, 112902. DOI. ADS.

Lemen, J.R., Title, A.M., Akin, D.J., Boerner, P.F., Chou, C., Drake, J.F., Duncan, D.W., Edwards, C.G., Friedlaender, F.M., Heyman, G.F., Hurlburt, N.E., Katz, N.L., Kushner, G.D., Levay, M., Lindgren, R.W., Mathur, D.P., McFeaters, E.L., Mitchell, S., Rehse, R.A., Schrijver, C.J., Springer, L.A., Stern, R.A., Tarbell, T.D., Wuelser, J.-P., Wolfson, C.J., Yanari, C., Bookbinder, J.A., Cheimets, P.N., Caldwell, D., Deluca, E.E., Gates, R., Golub, L., Park, S., Podgorski, W.A., Bush, R.I., Scherrer, P.H., Gummin, M.A., Smith, P., Auker, G., Jerram, P., Pool, P., Soufli, R., Windt, D.L., Beardsley, S., Clapp, M., Lang, J., Waltham, N.: 2012, The Atmospheric Imaging Assembly (AIA) on the Solar Dynamics Observatory (SDO). Solar Phys. 275, 17. DOI. ADS.

Li, D., Yuan, D., Su, Y.N., Zhang, Q.M., Su, W., Ning, Z.J.: 2018, Non-damping oscillations at flaring loops. Astron. Astrophys. 617, A86. DOI. ADS.

Magyar, N., Nakariakov, V.M.: 2020, Standing kink waves in sigmoid solar coronal loops: implications for coronal seismology. Astrophys. J. Lett. 894, L23. DOI. ADS.

Martens, P.C.H.: 2010, Scaling laws and temperature profiles for solar and stellar coronal loops with nonuniform heating. Astrophys. J. 714, 1290. DOI. ADS.

Müller, D., St. Cyr, O.C., Zouganelis, I., Gilbert, H.R., Marsden, R., Nieves-Chinchilla, T., Antonucci, E., Auchère, F., Berghmans, D., Horbury, T.S., Howard, R.A., Krucker, S., Maksimovic, M., Owen, C.J., Rochus, P., Rodriguez-Pacheco, J., Romoli, M., Solanki, S.K., Bruno, R., Carlsson, M., Fludra, A., Harra, L., Hassler, D.M., Livi, S., Louarn, P., Peter, H., Schühle, U., Teriaca, L., del Toro Iniesta, J.C., Wimmer-Schweingruber, R.F., Marsch, E., Velli, M., De Groof, A., Walsh, A., Williams, D.: 2020, The Solar Orbiter mission. Science overview. Astron. Astrophys. 642, A1. DOI. ADS.

Nakariakov, V.M., Kolotkov, D.Y.: 2020, Magnetohydrodynamic waves in the solar corona. Annu. Rev. Astron. Astrophys. 58, 441. DOI. ADS. 
Nakariakov, V.M., Anfinogentov, S.A., Nisticò, G., Lee, D.-H.: 2016, Undamped transverse oscillations of coronal loops as a self-oscillatory process. Astron. Astrophys. 591, L5. DOI. ADS.

Pascoe, D.J., Hood, A.W., Van Doorsselaere, T.: 2019, Coronal loop seismology using standing kink oscillations with a lookup table. Front. Astron. Space Sci. 6, 22. DOI. ADS.

Pascoe, D.J., Goddard, C.R., Nisticò, G., Anfinogentov, S., Nakariakov, V.M.: 2016, Coronal loop seismology using damping of standing kink oscillations by mode coupling. Astron. Astrophys. 589, A136. DOI. ADS.

Pascoe, D.J., Anfinogentov, S., Nisticò, G., Goddard, C.R., Nakariakov, V.M.: 2017, Coronal loop seismology using damping of standing kink oscillations by mode coupling. II. Additional physical effects and Bayesian analysis. Astron. Astrophys. 600, A78. DOI. ADS.

Rochus, P., Auchère, F., Berghmans, D., Harra, L., Schmutz, W., Schühle, U., Addison, P., Appourchaux, T., Aznar Cuadrado, R., Baker, D., Barbay, J., Bates, D., BenMoussa, A., Bergmann, M., Beurthe, C., Borgo, B., Bonte, K., Bouzit, M., Bradley, L., Büchel, V., Buchlin, E., Büchner, J., Cabé, F., Cadiergues, L., Chaigneau, M., Chares, B., Choque Cortez, C., Coker, P., Condamin, M., Coumar, S., Curdt, W., Cutler, J., Davies, D., Davison, G., Defise, J.-M., Del Zanna, G., Delmotte, F., Delouille, V., Dolla, L., Dumesnil, C., Dürig, F., Enge, R., François, S., Fourmond, J.-J., Gillis, J.-M., Giordanengo, B., Gissot, S., Green, L.M., Guerreiro, N., Guilbaud, A., Gyo, M., Haberreiter, M., Hafiz, A., Hailey, M., Halain, J.-P., Hansotte, J., Hecquet, C., Heerlein, K., Hellin, M.-L., Hemsley, S., Hermans, A., Hervier, V., Hochedez, J.-F., Houbrechts, Y., Ihsan, K., Jacques, L., Jérôme, A., Jones, J., Kahle, M., Kennedy, T., Klaproth, M., Kolleck, M., Koller, S., Kotsialos, E., Kraaikamp, E., Langer, P., Lawrenson, A., Le Clech', J.-C., Lenaerts, C., Liebecq, S., Linder, D., Long, D.M., Mampaey, B., Markiewicz-Innes, D., Marquet, B., Marsch, E., Matthews, S., Mazy, E., Mazzoli, A., Meining, S., Meltchakov, E., Mercier, R., Meyer, S., Monecke, M., Monfort, F., Morinaud, G., Moron, F., Mountney, L., Müller, R., Nicula, B., Parenti, S., Peter, H., Pfiffner, D., Philippon, A., Phillips, I., Plesseria, J.-Y., Pylyser, E., Rabecki, F., Ravet-Krill, M.-F., Rebellato, J., Renotte, E., Rodriguez, L., Roose, S., Rosin, J., Rossi, L., Roth, P., Rouesnel, F., Roulliay, M., Rousseau, A., Ruane, K., Scanlan, J., Schlatter, P., Seaton, D.B., Silliman, K., Smit, S., Smith, P.J., Solanki, S.K., Spescha, M., Spencer, A., Stegen, K., Stockman, Y., Szwec, N., Tamiatto, C., Tandy, J., Teriaca, L., Theobald, C., Tychon, I., van Driel-Gesztelyi, L., Verbeeck, C., Vial, J.-C., Werner, S., West, M.J., Westwood, D., Wiegelmann, T., Willis, G., Winter, B., Zerr, A., Zhang, X., Zhukov, A.N.: 2020, The Solar Orbiter EUI instrument: the Extreme Ultraviolet Imager. Astron. Astrophys. 642, A8. DOI. ADS.

Ruderman, M.S., Petrukhin, N.S.: 2021, Excitation of decayless kink oscillations by random motion. Mon. Not. Roy. Astron. Soc. 501, 3017. DOI. ADS.

Selesnick, I.W., Baraniuk, R.G., Kingsbury, N.C.: 2005, The dual-tree complex wavelet transform. IEEE Signal Process. Mag. 22, 123. DOI. ADS.

Shi, M., Van Doorsselaere, T., Guo, M., Karampelas, K., Li, B., Antolin, P.: 2021, The first 3D coronal loop model heated by MHD waves against radiative losses. Astrophys. J. 908, 233. DOI. ADS.

Tian, H., McIntosh, S.W., Wang, T., Ofman, L., De Pontieu, B., Innes, D.E., Peter, H.: 2012, Persistent Doppler shift oscillations observed with Hinode/EIS in the solar corona: spectroscopic signatures of Alfvénic waves and recurring upflows. Astrophys. J. 759, 144. DOI. ADS.

Van Doorsselaere, T., Brady, C.S., Verwichte, E., Nakariakov, V.M.: 2008, Seismological demonstration of perpendicular density structuring in the solar corona. Astron. Astrophys. 491, L9. DOI. ADS.

Van Doorsselaere, T., Srivastava, A.K., Antolin, P., Magyar, N., Vasheghani Farahani, S., Tian, H., Kolotkov, D., Ofman, L., Guo, M., Arregui, I., De Moortel, I., Pascoe, D.: 2020, Coronal heating by MHD waves. Space Sci. Rev. 216, 140. DOI. ADS.

Wadhwa, N., Rubinstein, M., Durand, F., Freeman, W.T.: 2013, Phase-based video motion processing. ACM Trans. Graph. 32, 80:1. DOI.

Wang, T., Ofman, L., Davila, J.M., Su, Y.: 2012, Growing transverse oscillations of a multistranded loop observed by SDO/AIA. Astrophys. J. Lett. 751, L27. DOI. ADS.

Williams, T., Walsh, R.W., Winebarger, A.R., Brooks, D.H., Cirtain, J.W., De Pontieu, B., Golub, L., Kobayashi, K., McKenzie, D.E., Morton, R.J., Peter, H., Rachmeler, L.A., Savage, S.L., Testa, P., Tiwari, S.K., Warren, H.P., Watkinson, B.J.: 2020, Is the high-resolution coronal imager resolving coronal strands? Results from AR 12712. Astrophys. J. 892, 134. DOI. ADS.

Wu, H.Y., Rubinstein, M., Shih, E., Guttag, J., Durand, F., Freeman, W.: 2012, Eulerian video magnification for revealing subtle changes in the world. ACM Trans. Graph. 31, 1. DOI.

Publisher's Note Springer Nature remains neutral with regard to jurisdictional claims in published maps and institutional affiliations. 